\title{
Current Management of Type 2 Diabetes Mellitus in Primary Care Clinics in Korea
}

\author{
Da Hea Seo ${ }^{1,2, *}$, Shinae Kang ${ }^{3,4, *}$, Yong-ho Lee ${ }^{2,5,6}$, Jung Yoon $\mathrm{Ha}^{7}$, Jong Suk Park ${ }^{3,4}$, Byoung-Wan Lee, ${ }^{2,5,6}$, \\ Eun Seok Kang ${ }^{2,5,6}$, Chul Woo Ahn ${ }^{3,4}$, Bong-Soo Cha ${ }^{2,5,6}$ \\ ${ }^{1}$ Department of Endocrinology and Metabolism, Inha University School of Medicine, Incheon; ${ }^{2}$ Graduate School, Yonsei \\ University College of Medicine; ${ }^{3}$ Department of Internal Medicine, Gangnam Severance Hospital, Yonsei University College of \\ Medicine; ${ }^{4}$ Severance Institute for Vascular and Metabolic Research, ${ }^{5}$ Division of Endocrinology and Metabolism, Department \\ of Internal Medicine, ${ }^{6}$ Institute of Endocrine Research, Yonsei University College of Medicine; ${ }^{7}$ Medical Information Team, \\ Ildong Pharmaceutical Co., Ltd., Seoul, Korea
}

Background: This study investigated the overall status of diabetes control and screening for diabetic microvascular complications in patients with type 2 diabetes mellitus attending primary care clinics in Korea.

Methods: In this cross-sectional observational study, 191 primary care clinics were randomly selected across Korea from 2015 to 2016. In total, 3,227 subjects were enrolled in the study.

Results: The patients followed at the primary care clinics were relatively young, with a mean age of $61.4 \pm 11.7$ years, and had a relatively short duration of diabetes (mean duration, $7.6 \pm 6.5$ years). Approximately $14 \%$ of subjects had diabetic microvascular complications. However, the patients treated at the primary care clinics had suboptimal control of hemoglobin A1c levels, blood pressure, and serum lipid levels, along with a metabolic target achievement rate of $5.9 \%$ according to the Korean Diabetes Association guidelines. The screening rates for diabetic nephropathy, retinopathy, and neuropathy within the past 12 months were $28.4 \%, 23.3 \%$, and $13.3 \%$, respectively.

Conclusion: The overall status of diabetes management, including the frequency of screening for microvascular complications, was suboptimal in the primary care clinics. More efforts should be made and more resources need to be allocated for primary care physicians to promote adequate healthcare delivery, which would result in stricter diabetes control and improved management of diabetic complications.

Keywords: Diabetes mellitus, type 2; Diabetes complications; Prevention and control; Primary health care; Tertiary care centers

\section{INTRODUCTION}

The prevalence of type 2 diabetes mellitus (T2DM) continues to rise and is projected to increase to 592 million in 2035 world-

Received: 22 April 2019, Revised: 19 July 2019, Accepted: 30 July 2019

Corresponding author: Bong-Soo Cha

Division of Endocrinology and Metabolism, Department of Internal Medicine, Endocrine Research Institute, Yonsei University College of Medicine, 50-1 Yonsei-ro, Seodaemun-gu, Seoul 03722, Korea

Tel: +82-2-2228-1962, Fax: +82-2-393-6884, E-mail: BSCHA@yuhs.ac

*These authors contributed equally to this work. wide [1,2]. Unfortunately, Korea is not an exception; the prevalence of diabetes is steadily increasing and a recent study revealed that about 4.8 million Korean adults $(13.7 \%)$ have diabetes [3]. Both microvascular and macrovascular complications

Copyright $\odot 2019$ Korean Endocrine Society

This is an Open Access article distributed under the terms of the Creative Commons Attribution Non-Commercial License (http://creativecommons.org/ licenses/by-nc/4.0/) which permits unrestricted non-commercial use, distribution, and reproduction in any medium, provided the original work is properly cited. 
develop quite commonly in patients with diabetes, and cause significant morbidity and mortality $[4,5]$.

Trials investigating diabetes control and complications have shown that intensive glycemic treatment effectively delays the onset and progression of diabetic microvascular complications in type 1 diabetes [6], and similar findings were noted in patients with T2DM [7], with benefits extending beyond the period of intervention [8,9]. Despite strong evidence of the benefits of tight glycemic control, an analysis of data from the 2014 Korea National Health and Nutrition Examination Survey (KNHANES) indicated that more than $50 \%$ of adults diagnosed with diabetes in Korea failed to achieve a hemoglobin A1c (HbAlc) level below $7 \%$, a result similar to that found in the USA $[10,11]$. A few studies have demonstrated that the overall diabetes management in patients who attended primary care clinics was less optimal than that of patients followed at secondary or tertiary hospitals [12-14], but there is a paucity of data comparing primary care to secondary or tertiary care following the release of the Korean Diabetes Association (KDA) clinical practice guidelines.

In response to the increasing prevalence of non-communicable diseases (NCDs), the Korean government introduced a clinic-centered NCD management policy in 2012, which focused on strengthening the role of primary care clinics as an important source of outpatient care for NCDs [15]. According to the Health Insurance Review \& Assessment Service, approximately $60 \%$ of patients with diabetes visited primary care clinics for management of diabetes in 2017 [16]; however, very few studies have examined the rate of adherence to the KDA guidelines in primary care clinics. The primary aim of this study was to examine the current status of overall diabetes management, including the screening rate for microvascular complications, among patients with T2DM visiting primary care clinics. The secondary aim was to compare the findings from primary care clinics to those from two tertiary hospitals in Seoul.

\section{METHODS}

\section{Study design and population}

A total of 191 primary care clinics were randomly selected across Korea, from which 2,915 patients with T2DM were enrolled in this study from January 2015 to September 2016 (Fig. 1). To maximize the generalizability of the results, the subjects were selected by random sampling from 16 cities and provinces (seven special and metropolitan cities including Seoul, Busan, Daegu, Incheon, Gwangju, Daejeon, and Ulsan) and nine provinces (Gyeonggi-do, Gangwon-do, Chungcheongbuk-do, Chungcheongnam-do, Jeollabuk-do, Jeollanam-do, Gyeongsangbukdo, Gyeongsangnam-do, and Jeju provinces) across Korea. The regional distribution of the enrollees was similar to that of the entire Korean population, as in the 2017 Korean Population Census [17]. As a secondary objective of our study, we compared primary care clinics with tertiary hospitals by analyzing

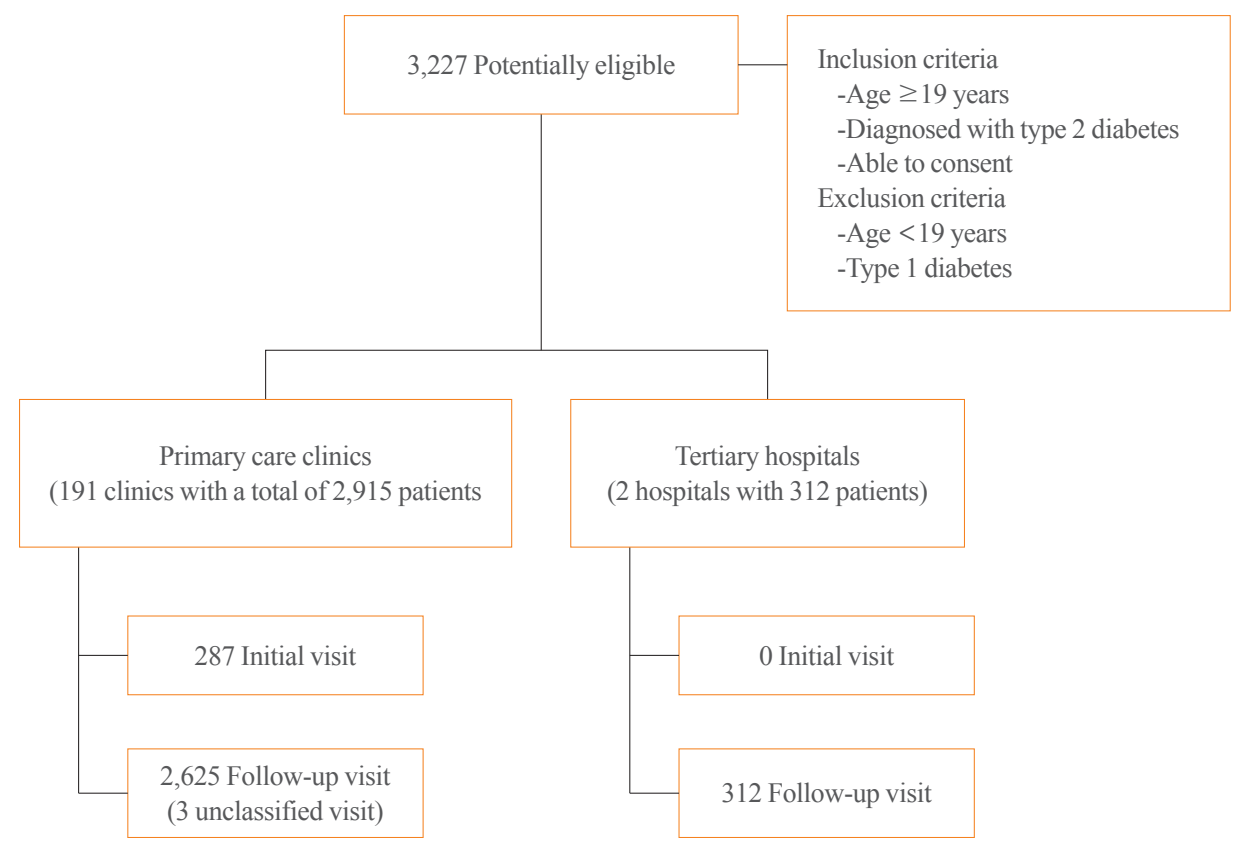

Fig. 1. Flowchart of the patients enrolled in the study. 
data from 312 patients with T2DM who attended tertiary hospitals for diabetes management, at Severance Hospital in the northern part of Seoul and Gangnam Severance Hospital in the southern part of Seoul (the total number of outpatient visits for diabetes management per year are 30,879 patients/year and 16,155 patients/year at Severance Hospital and Gangnam Severance Hospital, respectively). Among all patients who met both inclusion and exclusion criteria, those who consented were enrolled in the study consecutively. The inclusion criteria of the study were patients with T2DM who were $\geq 19$ years old and were willing to provide consent. Patients with type 1 diabetes and patients who were $<19$ years old were excluded. A diagnosis of type 1 diabetes were made if one of the following conditions was present; fasting C-peptide $\leq 0.6 \mathrm{ng} / \mathrm{mL}$, postprandial 2-hour C-peptide $\leq 1.8 \mathrm{ng} / \mathrm{mL}$, anti- glutamic acid decarboxylase antibody positivity, anti-insulin antibody positivity, or antiislet cell antibody positivity. Atherosclerotic cardiovascular disease (ASCVD) was defined as a previous history of stroke, ischemic heart disease, or peripheral vascular disease. Data, including laboratory assessments, were collected on the day of the outpatient clinic visit and were recorded in a pre-formulated case report by the physicians. The study protocol was approved by the Institutional Review Board of Severance Hospital, and written informed consent was obtained from all participants (IRB no. 4-2014-0904 and IRB no. 3-2014-0270).

\section{Anthropometric and biochemical measurements}

Data collected from the patients included demographic characteristics, past medical history, duration of diabetes, smoking history, as well as any history of diabetic vascular complications within the 12 months before enrollment. The treatment modalities of diabetes were classified as diet only, oral hypoglycemic agents (OHAs) alone, insulin alone, or a combination of insulin and OHAs. For those who were current smokers or had any history of smoking, data on whether they were ever offered smoking cessation intervention during previous encounters with the physicians were also gathered.

Each patient's height, weight, waist circumference, and blood pressure (BP) were measured and participants were also asked about the date and modality of BP measurements (i.e., digital method or analog method). Hypertension was defined as a BP $\geq 140 / 90 \mathrm{~mm} \mathrm{Hg}$ or taking antihypertensive medication(s).

Data on when the laboratory assessments were done were also collected, as well as the types of blood tests (capillary or plasma blood test) and the frequency and type of glycemic assessments (fasting glucose, random glucose, or $\mathrm{HbA1c}$ ). To evaluate dys- lipidemia, data were collected on the types of lipid tests (total lipid panel or partial lipid profile) in the 12 months before enrollment. Screening for microvascular complications was considered to have been done if there were records of any of the following: fundus eye exam, spot urine test for albumin-to-creatinine ratio or 24-hour urine test, monofilament test, pinprick test, ankle reflex test, nerve conduction test, or a referral to a specialist within the previous 12 to 24 months. Microalbuminuria and overt proteinuria were defined as a random urine albumin-tocreatinine ratio between 30 and $300 \mathrm{mg} / \mathrm{g}$ and above $300 \mathrm{mg} / \mathrm{g}$, respectively. The prevalence of retinopathy, nephropathy, neuropathy, ischemic heart disease, cerebrovascular disease, and peripheral artery disease was investigated based on the patient's past medical history. The optimal targets were defined as $\mathrm{HbA1c}$ $<6.5 \%$ for blood glucose, $<140 / 85 \mathrm{~mm} \mathrm{Hg}$ for BP, and low density lipoprotein cholesterol (LDL-C) $<100 \mathrm{mg} / \mathrm{dL}$ for the blood lipid profile [18]. For those with ASCVD, BP $<130 / 80 \mathrm{~mm} \mathrm{Hg}$ and serum LDL-C $<70 \mathrm{mg} / \mathrm{dL}$ were defined as the optimal targets according to the KDA treatment guideline for diabetes 2019 [18].

\section{Statistical analysis}

We used SPSS version 20.0 (IBM Corp., Armonk, NY, USA) for the statistical analysis and considered $P$ values $<0.05$ to indicate statistical significance. Continuous variables with a normal distribution were presented as mean \pm standard deviation (SD), and categorical variables as absolute numbers (percentages). The Student $t$ test, chi-square test, and Fisher exact test were used to test the statistical significance of differences between mean values.

\section{RESULTS}

\section{General characteristics and diabetes control status of the study population}

The mean age of the subjects in the primary care setting was $61.4 \pm 11.7$ years and the mean duration of diabetes was 7.6 6.5 years, with a mean $\mathrm{HbA} 1 \mathrm{c}$ of $7.6 \% \pm 2.0 \%$. Approximately two-thirds of the patients had concomitant hypertension and dyslipidemia, with suboptimal control of blood glucose, serum lipids, and BP (Table 1). The prevalence of diabetic microvascular and macrovascular complications were $13.7 \%$ and $14.4 \%$, respectively (Table 1). Among the 2,915 patients with T2DM followed at primary care clinics, the majority of the patients were treated with OHAs $(96 \%)$ and the most frequently prescribed OHA was metformin (84\%), followed by dipeptidyl 
Table 1. General Characteristics of the Study Population

\begin{tabular}{|c|c|c|c|}
\hline Characteristic & Primary $(n=2,915)$ & Tertiary $(n=312)$ & $P$ value \\
\hline Age, yr & $61.4 \pm 11.7$ & $63.4 \pm 11.0$ & $0.004^{\mathrm{a}}$ \\
\hline Male sex & $1,612(55.3)$ & $189(60.6)$ & 0.075 \\
\hline Age at diabetes diagnosis, yr & $53.8 \pm 11.3$ & $53.3 \pm 10.9$ & 0.484 \\
\hline Duration of diabetes, $\mathrm{yr}$ & $7.6 \pm 6.5$ & $10.1 \pm 8.7$ & $<0.0001^{\mathrm{a}}$ \\
\hline \multicolumn{4}{|l|}{ Visit type } \\
\hline Initial visit & $287(9.9)$ & 0 & $<0.0001^{\mathrm{a}}$ \\
\hline Follow-up visit & $2,625(90.1)$ & $312(100.0)$ & $<0.0001^{\mathrm{a}}$ \\
\hline BMI, $\mathrm{kg} / \mathrm{m}^{2}$ & $25.1 \pm 3.4$ & $25.3 \pm 3.3$ & 0.377 \\
\hline Male & $25.2 \pm 3.2$ & $25.2 \pm 3.4$ & 0.957 \\
\hline Female & $25.0 \pm 3.7$ & $25.4 \pm 3.1$ & 0.184 \\
\hline Weight, kg & $67.5 \pm 12.1$ & $67.8 \pm 11.6$ & 0.673 \\
\hline Male & $72.8 \pm 11.1$ & $72.0 \pm 11.0$ & 0.397 \\
\hline Female & $60.8 \pm 9.7$ & $61.2 \pm 9.1$ & 0.712 \\
\hline $\mathrm{WC}, \mathrm{cm}$ & $86.6 \pm 9.6$ & $88.5 \pm 8.2$ & 0.100 \\
\hline Male & $88.6 \pm 8.7$ & $89.0 \pm 9.6$ & 0.600 \\
\hline Female & $83.8 \pm 10.1$ & $85.3 \pm 9.0$ & 0.140 \\
\hline Hypertension & $1,718(58.9)$ & $160(51.4)$ & $0.011^{\mathrm{a}}$ \\
\hline Dyslipidemia & $1,736(59.6)$ & $181(58.2)$ & 0.644 \\
\hline SBP, mm Hg & $128.3 \pm 12.9$ & $121.5 \pm 12.7$ & $<0.0001^{\mathrm{a}}$ \\
\hline DBP, $\mathrm{mm} \mathrm{Hg}$ & $78.2 \pm 8.9$ & $73.4 \pm 9.7$ & $<0.0001^{\mathrm{a}}$ \\
\hline $\mathrm{HbA1c}, \%$ & $7.6 \pm 2.0$ & $6.9 \pm 0.9$ & $<0.0001^{\mathrm{a}}$ \\
\hline Fasting glucose, mg/dL & $145.9 \pm 56.1$ & $127.4 \pm 28.0$ & $<0.0001^{\mathrm{a}}$ \\
\hline Total cholesterol, mg/dL & $177.2 \pm 41.8$ & $157.0 \pm 31.3$ & $<0.0001^{\mathrm{a}}$ \\
\hline $\mathrm{TG}, \mathrm{mg} / \mathrm{dL}$ & $166.4 \pm 102.1$ & $136.9 \pm 104.2$ & $<0.0001^{\mathrm{a}}$ \\
\hline $\mathrm{HDL}-\mathrm{C}, \mathrm{mg} / \mathrm{dL}$ & $48.3 \pm 13.0$ & $47.5 \pm 11.5$ & 0.265 \\
\hline LDL-C, mg/dL & $98.3 \pm 34.8$ & $83.2 \pm 26.3$ & $<0.0001^{\mathrm{a}}$ \\
\hline Creatinine, $\mathrm{mg} / \mathrm{dL}$ & $1.2 \pm 4.4$ & $0.9 \pm 0.4$ & $0.002^{\mathrm{a}}$ \\
\hline UACR, $\mathrm{mg} / \mathrm{g}$ & $80.0 \pm 332.1$ & $68.7 \pm 298.5$ & 0.597 \\
\hline Current smoker & $603(20.7)$ & $37(11.9)$ & $<0.0001^{\mathrm{a}}$ \\
\hline Smoking cessation intervention ${ }^{\mathrm{b}}$ & $772(84.6)$ & $68(43.0)$ & $<0.0001^{\mathrm{a}}$ \\
\hline Microvascular complications $^{\mathrm{c}}$ & $399(13.7)$ & $79(25.4)$ & $<0.0001^{\mathrm{a}}$ \\
\hline Diabetic retinopathy & $132(4.5)$ & $49(15.8)$ & $<0.0001^{\mathrm{a}}$ \\
\hline Diabetic nephropathy & $141(4.8)$ & $28(9.0)$ & $0.002^{\mathrm{a}}$ \\
\hline Diabetic neuropathy & $171(5.9)$ & $27(8.7)$ & $0.049^{\mathrm{a}}$ \\
\hline Macrovascular complications ${ }^{c}$ & $421(14.4)$ & $58(18.6)$ & 0.050 \\
\hline OHA alone & $2,695(92.5)$ & $278(89.1)$ & $0.037^{\mathrm{a}}$ \\
\hline Monotherapy ${ }^{d}$ & $670(24.9)$ & $116(41.7)$ & $<0.0001^{\mathrm{a}}$ \\
\hline Dual therapy ${ }^{d}$ & $1,361(50.5)$ & $124(44.6)$ & $0.008^{\mathrm{a}}$ \\
\hline Triple therapy ${ }^{\mathrm{d}}$ & $692(25.7)$ & $64(23.0)$ & 0.145 \\
\hline OHA (alone or with insulin) & $2,795(95.9)$ & $305(97.8)$ & 0.106 \\
\hline Insulin & $114(3.9)$ & $29(9.3)$ & $<0.0001^{\mathrm{a}}$ \\
\hline Insulin alone $\mathrm{e}^{\mathrm{e}}$ & $14(12.3)$ & $2(6.9)$ & 0.527 \\
\hline Insulin with $\mathrm{OHA}^{\mathrm{e}}$ & $100(87.7)$ & $27(93.1)$ & 0.527 \\
\hline No medication & $106(3.6)$ & $5(1.6)$ & 0.061 \\
\hline
\end{tabular}

Values are expressed as mean \pm standard deviation or number (\%). $P$ values refer to the unpaired $t$ test or the chi-square test.

BMI, body mass index; WC, waist circumference; SBP, systolic blood pressure; DBP, diastolic blood pressure; HbA1c; hemoglobin A1c; TG, triglyceride; HDL$\mathrm{C}$, high density lipoprotein cholesterol; LDL-C, low density lipoprotein cholesterol; UACR, urinary albumin-to-creatinine ratio; OHA, oral hypoglycemic agent. ${ }^{a}$ Values with statistical significance; ${ }^{b}$ The denominator is the total number of current smokers and those who had a history of smoking after the diagnosis of diabetes; ${ }^{\circ}$ Excludes those with multiple choices/answers; ${ }^{\mathrm{d}}$ The denominator is the number of people taking OHAs only; ${ }^{\mathrm{e}}$ The denominator is the total number of people taking insulin with or without OHAs. 
peptidase 4 (DPP4) inhibitors (65\%) and sulfonylureas (41\%) (Supplemental Table S1). The proportion of the patients prescribed OHAs was significantly higher than the insulin prescrip-

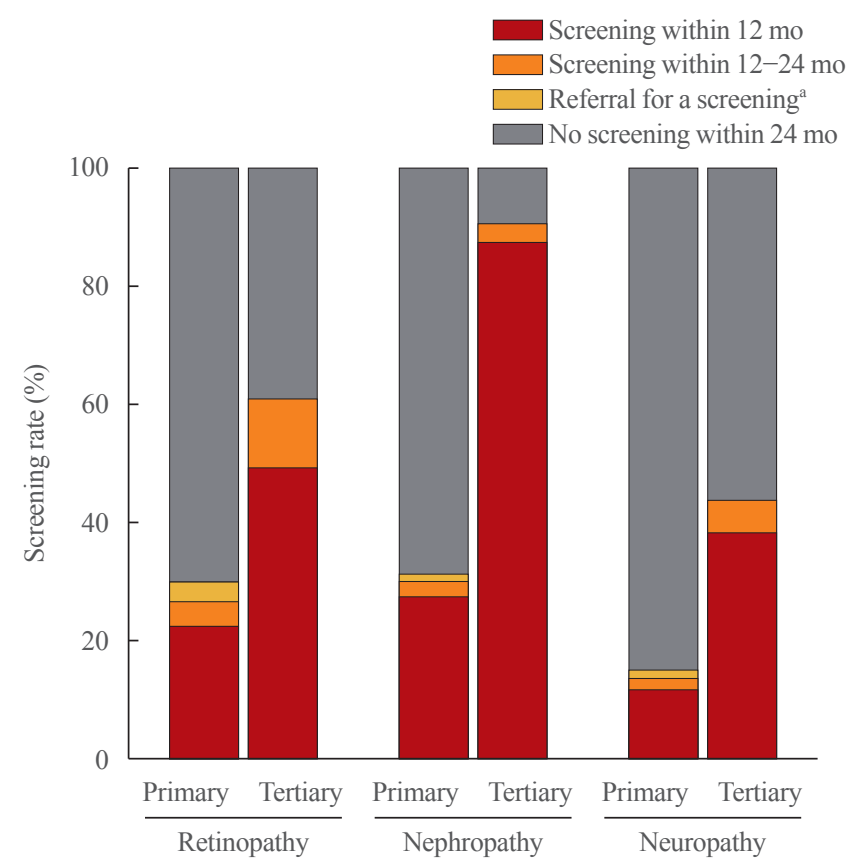

Fig. 2. Comparison of the screening done for microvascular complications at primary care clinics versus the tertiary hospitals. ${ }^{a}$ Referral for screening tests within 24 months.

Table 2. Screening for Diabetic Microvascular Complications

\begin{tabular}{lcc}
\hline Variable & $\begin{array}{c}\text { Primary } \\
(n=2,915)\end{array}$ & $\begin{array}{c}\text { Tertiary } \\
(n=312)\end{array}$ \\
\hline Diabetic retinopathy & $679(23.3)$ & $155(49.7)$ \\
Within 12 mo & $114(3.9)$ & $37(11.9)$ \\
12-24 mo & $114(3.9)$ & 0 \\
Referral for screening tests within 24 mo & $2,008(68.9)$ & $120(38.5)$ \\
No tests within 24 mo & & \\
Diabetic nephropathy & $828(28.4)$ & $274(87.8)$ \\
Within 12 mo & $77(2.6)$ & $10(3.2)$ \\
12-24 mo & $32(1.1)$ & 0 \\
Referral for screening tests within 24 mo & $1,977(67.8)$ & $28(9.0)$ \\
No tests within 24 mo & & \\
Diabetic neuropathy & $388(13.3)$ & $122(39.1)$ \\
Within 12 mo & $43(1.5)$ & $17(5.4)$ \\
12-24 mo & $44(1.5)$ & 0 \\
Referral for screening tests within 24 mo & $2,440(83.7)$ & $173(55.4)$ \\
No tests within 24 mo & & \\
\hline Values are expressed as number (\%). & & \\
\hline
\end{tabular}

tion rate of $4 \%$ (Table 1$)$.

\section{Screening rate for diabetic microvascular complications and metabolic target achievement rate}

The overall prevalence of diabetic complications was significantly lower among the patients treated at primary care clinics than among those treated at tertiary hospitals (Table 1). The screening rates for diabetic retinopathy, nephropathy, and neuropathy within the past 12 months were $23 \%, 28 \%$, and $13 \%$, respectively, at primary care clinics (Table 2, Fig. 2). In other words, more than two-thirds of patients did not receive any screening tests in a year. Moreover, the proportion of the patients who achieved optimal metabolic targets (HbA1c, BP, and LDL-C) was only $5.9 \%$ among those followed at primary care clinics (Table 3). In a sub-analysis of those with ASCVD, the proportion of subjects who achieved the optimal BP and lipid profile targets (BP <130/80 mm Hg and LDL-C $<70 \mathrm{mg} / \mathrm{dL}$ ) was $26.4 \%$ and $16.6 \%$, respectively (Supplemental Table S2).

\begin{tabular}{lccc} 
Table 3. Metabolic Target Achievement Rate & \\
\hline Variable & $\begin{array}{c}\text { Primary } \\
(n=2,915)\end{array}$ & $\begin{array}{c}\text { Tertiary } \\
(n=312)\end{array}$ & $P$ value \\
\hline Glycemic control & $501(22.5)$ & $105(34.4)$ & $<0.0001^{\mathrm{a}}$ \\
HbA1c $<6.5 \%^{\mathrm{b}}$ & $934(41.9)$ & $193(63.3)$ & $<0.0001^{\mathrm{a}}$ \\
HbA1c $<7.0 \%{ }^{\mathrm{b}}$ & & & \\
BP control & $1,913(65.6)$ & $257(82.4)$ & $<0.0001^{\mathrm{a}}$ \\
BP $<140 / 85 \mathrm{~mm} \mathrm{Hg}$ & $2,057(70.6)$ & $307(98.4)$ & $<0.0001^{\mathrm{a}}$ \\
BP $<140 / 90 \mathrm{~mm} \mathrm{Hg}$ & & & \\
Dyslipidemia control & $1,132(55.7)$ & $239(78.6)$ & $<0.0001^{\mathrm{a}}$ \\
LDL-C $<100 \mathrm{mg} / \mathrm{dL}^{\mathrm{c}}$ & $1,645(73.8)$ & $224(73.4)$ & 0.884 \\
HDL-C $\geq 40 \mathrm{mg} / \mathrm{dL}^{\mathrm{d}}$ & $1,290(54.5)$ & $216(70.6)$ & $<0.0001^{\mathrm{a}}$ \\
TG $<150 \mathrm{mg} / \mathrm{dL}^{\mathrm{e}}$ & $172(5.9)$ & $67(21.5)$ & $<0.0001^{\mathrm{a}}$ \\
Three metabolic targets & & &
\end{tabular}

Values are expressed as number (\%). $P$ values refer to the chi-square test.

HbA1c, hemoglobin A1c; BP, blood pressure; LDL-C, low density lipoprotein cholesterol; HDL-C, high density lipoprotein cholesterol; TG, triglyceride.

${ }^{a}$ Values with statistical significance; ${ }^{b}$ The denominator is the number of people who had $\mathrm{HbA} 1 \mathrm{c}$ testing within 3 months prior to enrollment $(n=2,224)$; ${ }^{\mathrm{c}}$ The denominator is the number of people who had LDL-C testing within 12 months prior to enrollment $(n=2,031)$; ${ }^{\mathrm{d}}$ The denominator is the number of people who had HDL-C testing within 12 months prior to enrollment $(n=2,228)$; ${ }^{\mathrm{e}}$ The denominator is the number of people who had TG testing within 12 months prior to enrollment $(n=2,366)$; ${ }^{\mathrm{f}}$ The denominator is the total number of subjects in each group; ${ }^{\mathrm{g}}$ Three metabolic targets: $\mathrm{HbA} 1 \mathrm{c}<6.5 \%$, BP $<140 / 85 \mathrm{~mm} \mathrm{Hg}$, and LDL-C $<100 \mathrm{mg} / \mathrm{dL}$. 


\section{Frequency and method of glucose, BP, and cholesterol monitoring}

Although $95 \%$ of the subjects had at least one form of glycemic assessment (random glucose testing, fasting glucose testing, or HbA1c test), HbAlc testing was significantly underutilized at primary care clinics. Furthermore, two-thirds of the patients followed at primary care clinics only received $\mathrm{HbAlc}$ tests one to two times per year (Table 4).

For BP monitoring, 94\% of the subjects had BP measurements taken on the day of their encounter at a primary care clinic, and the analog method was still preferred to assess BP. For lipid profile tests, the proportion of the subjects who had a total lipid panel test was significantly less at primary care clinics (Table 4).

\section{Smoking status and counseling}

Among the subjects who attended the primary care clinics, $20.7 \%$ were current smokers, and the majority of current smokers were offered smoking cessation education or counseling.

\section{DISCUSSION}

This study investigated the overall status of diabetes management in 2,915 patients with T2DM treated at primary care clinics in Korea, and found that most of the patients did not meet

Table 4. Frequency and Method of Tests for Diabetes Management

\begin{tabular}{|c|c|c|c|}
\hline Variable & Primary $(n=2,915)$ & Tertiary $(n=312)$ & $P$ value \\
\hline Glycemic tests $^{\mathrm{a}}$ & $2,743(94.1)$ & $311(99.7)$ & $<0.001^{\mathrm{b}}$ \\
\hline Fasting glucose test ${ }^{\mathrm{c}, \mathrm{d}}$ & $1,504(54.9)$ & $311(100.0)$ & $<0.001^{\mathrm{b}}$ \\
\hline Plasma blood sampling ${ }^{\mathrm{e}}$ & $756(50.3)$ & $311(100.0)$ & \\
\hline $\mathrm{POCT}^{\mathrm{P}}$ & $819(54.5)$ & 0 & \\
\hline Random glucose test ${ }^{\mathrm{c}, \mathrm{d}}$ & $1,638(59.7)$ & $20(6.4)$ & $<0.001^{\mathrm{b}}$ \\
\hline Plasma blood sampling ${ }^{\mathrm{f}}$ & $297(18.1)$ & $20(100.0)$ & \\
\hline POCT $^{f}$ & $1,352(82.5)$ & 0 & \\
\hline HbA1c test ${ }^{\mathrm{c}, \mathrm{d}}$ & $1,507(55.0)$ & $302(97.1)$ & $<0.001^{\mathrm{b}}$ \\
\hline Plasma blood sampling ${ }^{g}$ & $1,117(74.1)$ & $302(100.0)$ & \\
\hline $\mathrm{POCT}^{\mathrm{g}}$ & $391(25.9)$ & 0 & \\
\hline No. of $\mathrm{HbA} 1 \mathrm{c}$ tests within 12 months & & & $<0.0001^{\mathrm{b}}$ \\
\hline None & $191(6.6)$ & $1(0.3)$ & \\
\hline $1-2$ times/yr & $1,866(64.0)$ & $38(12.2)$ & \\
\hline$\geq 3$ times/yr & $706(24.2)$ & $273(87.5)$ & \\
\hline Did not know & $101(3.5)$ & 0 & \\
\hline Performing BP monitoring ${ }^{\text {h }}$ & $2,720(93.3)$ & $311(99.7)$ & $<0.0001^{\mathrm{b}}$ \\
\hline Analog method ${ }^{\mathrm{i}}$ & $1,726(63.5)$ & 0 & \\
\hline Digital method $^{\mathrm{i}}$ & $1,006(37.0)$ & $311(100.0)$ & \\
\hline Performing lipid profile test ${ }^{j}$ & $2,907(99.7)$ & $312(100.0)$ & $<0.0001^{\mathrm{b}}$ \\
\hline Total lipid panel ${ }^{\mathrm{k}}$ & $1,783(61.3)$ & $304(97.4)$ & \\
\hline Partial lipid test ${ }^{\mathrm{k}}$ (partial) & $696(23.9)$ & $8(2.6)$ & \\
\hline No lipid panel test ${ }^{\mathrm{k}}$ & $244(8.4)$ & 0 & \\
\hline
\end{tabular}

Values are expressed as number (\%). $P$ values refer to the chi-square test.

$\mathrm{POCT}$, point of care test; $\mathrm{HbA1c}$, hemoglobin A1c; BP, blood pressure.

${ }^{a}$ For glycemic control, patients were asked if they had blood tests on the day of the visit; ${ }^{b}$ Values with statistical significance; ${ }^{\circ}$ The denominator is the number of subjects who received a fasting glucose test; ${ }^{\mathrm{d}}$ Includes those with multiple choices/answers; ${ }^{\mathrm{e}}$ The denominator is the number of subjects who received a fasting glucose test; ${ }^{\mathrm{f}}$ The denominator is the number of subjects who received a random glucose test; ${ }^{\mathrm{g}}$ The denominator is the number of sub-

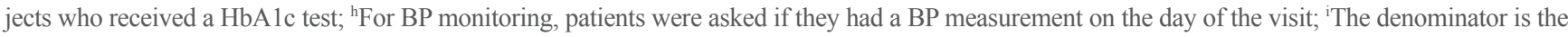
number of subjects who received BP monitoring on the day of visit; ${ }^{j}$ For the lipid profile test, patients attending an initial visit were asked if they had a blood test for their lipid profile on the day of the visit and patients attending a follow-up visit were asked if they had a blood test for their lipid profile within 12 months; ${ }^{k}$ The denominator is the number of subjects who received lipid profile tests within 12 months. 
the current clinical practice guidelines recommended by the KDA. Only approximately $22.5 \%$ of the patients achieved the recommended level of glycemic control. These findings agree with those of previous studies of primary care settings that showed a high frequency of poor glycemic control [12]. Our data also demonstrate that a low proportion of patients received screening tests for microvascular complications, which clearly demonstrates a deficiency in the management of diabetes. Hence, more efforts and suitable measures need to be introduced to improve the care provided to patients with diabetes at primary care clinics in Korea.

The prevalence of T2DM and its complications continues to rise $[10,19,20]$. In order to prevent diabetic microvascular and macrovascular complications, comprehensive management of diabetes is crucial. Despite the development of numerous new antidiabetic medications, the proportion of patients achieving target glycemic control (HbAlc $<7 \%$ defined by the American Diabetes Association [ADA] guidelines) has plateaued in the United States since 2006 [11]. In Korea, the proportion of the patients achieving optimal glycemic control (i.e., $\mathrm{HbAlc}<7 \%$,) continued to improve from $42.5 \%$ in 2005 to $44.9 \%$ in 2014 [10]; however, this proportion was significantly lower for patients treated at primary care clinics than for those treated at tertiary hospitals in our study $(41.9 \%$ vs. $63.3 \%, P<0.0001)$. In other words, a large proportion of patients are still at a high risk of developing diabetic complications, particularly those who attend the primary care clinics.

There have been significant changes in the prescription patterns of antidiabetic medications following the introduction of DPP4 inhibitors in 2008. In 2002, sulfonylureas were the most commonly used antidiabetic medications for T2DM patients; however, the prescription rate for metformin has steadily increased, and metformin has been the most frequently prescribed antidiabetic drug since 2013, followed by sulfonylureas and then DPP4 inhibitors [19]. These changes are clearly reflected in the prescription patterns found in our study, where the most commonly prescribed dual combination was metformin and a DPP4 inhibitor. DPP4 inhibitors are readily used by many healthcare providers, including primary care physicians, because of their ease of use, fair glycemic control, neutral effect on body weight, and low hypoglycemia rate. However, the proportion of the subjects prescribed insulin was very low at primary care clinics. This may be partly due to the introduction of various antidiabetic agents, including DPP4 inhibitors, and a shortage of educational staff with expertise on insulin therapy at primary care clinics [12].
Both ADA and KDA guidelines recommend monitoring $\mathrm{HbA1c}$ at least biannually in patients with stable glycemic control and quarterly in patients who do not meet their treatment goals or who have changed medication [18,21]. Despite steady improvements in the frequency of $\mathrm{HbAlc}$ monitoring since the introduction of a diabetes care quality assurance program [16], those managed at primary care clinics were infrequently tested for HbA1c [22]. Furthermore, consistent with previous reports $[16,22]$, random glucose testing using a portable glucometer was the most commonly used method for glycemic monitoring, most likely because of the lack of laboratory facilities at primary care clinics. This indicates that poor diabetes control in patients treated at primary care clinics may be related to less intensive and less frequent monitoring, despite shorter visit intervals.

Despite the recommendations on annual screening for diabetic microvascular complications contained in the KDA guideline [18], the screening rates for diabetic retinopathy, nephropathy, and neuropathy at primary care clinics were quite low. Previous studies have suggested that a lack of resources, such as limited access to facilities for screening tests for complications, and patients' socioeconomic status were associated with the frequency of diabetic screening tests [23-25]. Similar findings were also noted in the Pittsburgh Epidemiology of Diabetes Complications study, in which it was found that receiving care from primary care physicians, instead of from diabetes specialists, was associated with lower preventive service utilization and poorer glycemic control [26]. Physicians' attitudes toward caring for diabetic patients can be another barrier, as some primary care physicians felt that the guidelines for reaching the goals were not clear and relied on their clinical experience when making decisions to screen [24,27]. Moreover, they often faced administrative, time, and information constraints [27]. As preventive health care is the cornerstone of the primary and secondary prevention of diabetes complications, improving diabetes education for both patients and providers in primary care settings and establishing a system that allows easy referrals to specialists may improve the utilization of preventive services.

Moreover, a systemic program or a policy needs to be implemented by the government in order to improve and sustain the comprehensive management of diabetes at primary care clinics. It is necessary to introduce a compensation system based on the health outcomes of patients to encourage primary care physicians to participate more actively in the management of diabetes. For example, the introduction of non-monetary compensation systems, such as the Green Prescription Clinics, for clinics with excellent target achievement rates can drive voluntary 
quality improvement of primary care clinics.

There are some limitations of this study. First, this was a cross-sectional observational study, which did not allow us to monitor changes over time. Second, a total of 312 patients from two tertiary hospitals were enrolled for this study and used as comparators. However, the tertiary hospitals may have been primarily representative of the Gangbuk and Gangnam areas, whereas the primary endpoint of this study was to examine the current status of T2DM management at primary care clinics. Nevertheless, this is the first study to examine the current status of diabetes management in Koreans, especially with a focus on the frequency and method of follow-up tests and screenings for microvascular complications at primary care clinics.

In conclusion, despite steady improvements over the past decade, we found that the overall status of diabetes management, including the screening rates for complications, was still suboptimal in patients with T2DM who attended primary care clinics. Substantially more efforts and resources need to be deployed for primary physicians to improve their adherence to clinical practice guidelines and to deliver the most optimal healthcare in the primary care setting.

\section{CONFLICTS OF INTEREST}

This study was supported by a research grant from Ildong Pharmaceutical Co., Ltd. One of the co-authors, Jung Yoon Ha, is a current employee at Ildong Pharmaceutical Co., Ltd., but no potential conflict of interest was reported by the author.

\section{AUTHOR CONTRIBUTIONS}

Conception or design: S.K., Y.L., J.Y.H., B.S.C. Acquisition, analysis, or interpretation of data: D.H.S., S.K., Y.L., J.S.P., B.W.L., E.S.K., C.W.A., B.S.C. Drafting the work or revising: D.H.S., S.K., B.S.C. Final approval of the manuscript: D.H.S., S.K., B.S.C.

\section{ORCID}

Da Hea Seo https://orcid.org/0000-0003-2767-0293

Shinae Kang https://orcid.org/0000-0002-9719-4774

Bong-Soo Cha https://orcid.org/0000-0003-0542-2854

\section{REFERENCES}

1. Narayan KM, Boyle JP, Geiss LS, Saaddine JB, Thompson
TJ. Impact of recent increase in incidence on future diabetes burden: U.S., 2005-2050. Diabetes Care 2006;29:2114-6.

2. International Diabetes Federation. IDF diabetes atlas, 6th ed. Brussels: International Diabetes Federation; 2013.

3. Won JC, Lee JH, Kim JH, Kang ES, Won KC, Kim DJ, et al. Diabetes fact sheet in Korea, 2016: an appraisal of current status. Diabetes Metab J 2018;42:415-24.

4. UK Prospective Diabetes Study (UKPDS) Group. Intensive blood-glucose control with sulphonylureas or insulin compared with conventional treatment and risk of complications in patients with type 2 diabetes (UKPDS 33). Lancet 1998; 352:837-53.

5. Stratton IM, Adler AI, Neil HA, Matthews DR, Manley SE, Cull CA, et al. Association of glycaemia with macrovascular and microvascular complications of type 2 diabetes (UKPDS 35): prospective observational study. BMJ 2000;321: 405-12.

6. Diabetes Control and Complications Trial Research Group, Nathan DM, Genuth S, Lachin J, Cleary P, Crofford O, et al. The effect of intensive treatment of diabetes on the development and progression of long-term complications in insulindependent diabetes mellitus. N Engl J Med 1993;329:97786.

7. Duckworth W, Abraira C, Moritz T, Reda D, Emanuele N, Reaven PD, et al. Glucose control and vascular complications in veterans with type 2 diabetes. N Engl J Med 2009; 360:129-39.

8. Murray P, Chune GW, Raghavan VA. Legacy effects from DCCT and UKPDS: what they mean and implications for future diabetes trials. Curr Atheroscler Rep 2010;12:432-9.

9. Holman RR, Paul SK, Bethel MA, Neil HA, Matthews DR. Long-term follow-up after tight control of blood pressure in type 2 diabetes. N Engl J Med 2008;359:1565-76.

10. Ha KH, Kim DJ. Current status of managing diabetes mellitus in Korea. Korean J Intern Med 2016;31:845-50.

11. Carls G, Huynh J, Tuttle E, Yee J, Edelman SV. Achievement of glycated hemoglobin goals in the US remains unchanged through 2014. Diabetes Ther 2017;8:863-73.

12. Tai TY, Chuang LM, Tsai ST, Huang BY; Diabcare (Taiwan) Study Group. Treatment of type 2 diabetes mellitus in a primary care setting in Taiwan: comparison with secondary/ tertiary care. J Formos Med Assoc 2006;105:105-17.

13. Lim DJ, Kwon HS, Kim HS, Lee JH, Ko SH, Lee JM, et al. Clinical characteristics of the diabetic patients managed at the different medical institutions in Seoul and Gyeonggi province. Korean J Med 2006;71:173-81. 
14. Harris SB, Ekoe JM, Zdanowicz Y, Webster-Bogaert S. Glycemic control and morbidity in the Canadian primary care setting (results of the diabetes in Canada evaluation study). Diabetes Res Clin Pract 2005;70:90-7.

15. Park YH. Strategy for noncommunicable disease control and prevention. J Korean Med Assoc 2014;57:808-14.

16. Health Insurance Review \& Assessment Service. Comprehensive quality report on diabetes. Wonju: HIRA; 2017.

17. Statistics Korea. Population, households and housing units [Internet]. Daejeon: Statistics Korea; c1996 [cited 2019 Aug 16]. Available from: http://kostat.go.kr/portal/korea/index. action.

18. Korean Diabetes Association. Treatment guideline for diabetes. Seoul: KDA; 2019.

19. Ko SH, Han K, Lee YH, Noh J, Park CY, Kim DJ, et al. Past and current status of adult type 2 diabetes mellitus management in Korea: a National Health Insurance Service Database analysis. Diabetes Metab J 2018;42:93-100.

20. Korean Diabetes Association. Diabetes fact sheet in Korea 2018. Seoul: KDA; 2018.

21. Introduction: standards of medical care in diabetes. 2018. Diabetes Care 2018;41(Suppl 1):S1-2.

22. Yoo KH, Shin DW, Cho MH, Kim SH, Bahk HJ, Kim SH, et al. Regional variations in frequency of glycosylated hemoglobin (HbA1c) monitoring in Korea: a multilevel analysis of nationwide data. Diabetes Res Clin Pract 2017;131:61-9.

23. Liu Y, Zupan NJ, Shiyanbola OO, Swearingen R, Carlson JN, Jacobson NA, et al. Factors influencing patient adherence with diabetic eye screening in rural communities: a qualitative study. PLoS One 2018;13:e0206742.

24. van Eijk KN, Blom JW, Gussekloo J, Polak BC, Groeneveld Y. Diabetic retinopathy screening in patients with diabetes mellitus in primary care: incentives and barriers to screening attendance. Diabetes Res Clin Pract 2012;96:10-6.

25. Byun SH, Ma SH, Jun JK, Jung KW, Park B. Screening for diabetic retinopathy and nephropathy in patients with diabetes: a nationwide survey in Korea. PLoS One 2013;8:e62991.

26. Zgibor JC, Songer TJ, Kelsey SF, Weissfeld J, Drash AL, Becker D, et al. The association of diabetes specialist care with health care practices and glycemic control in patients with type 1 diabetes: a cross-sectional analysis from the Pittsburgh epidemiology of diabetes complications study. Diabetes Care 2000;23:472-6.

27. Zgibor JC, Songer TJ. External barriers to diabetes care: addressing personal and health systems issues. Diabetes Spectr 2001;14:23-8. 